\title{
In situ Observations of CIRs on STEREO, Wind, and ACE During 2007-2008
}

\author{
G.M. Mason • M.I. Desai • U. Mall • A. Korth • \\ R. Bucik · T.T. von Rosenvinge $\cdot$ K.D. Simunac
}

Received: 2 December 2008 / Accepted: 6 April 2009 / Published online: 17 April 2009

(C) The Author(s) 2009. This article is published with open access at Springerlink.com

\begin{abstract}
During the 2007 and 2008 solar minimum period, STEREO, Wind, and ACE observed numerous Corotating Interaction Regions (CIRs) over spatial separations that began with all the spacecraft close to Earth, through STEREO separation angles of $\sim 80$ degrees in the fall of 2008. Over 35 CIR events were of sufficient intensity to allow measurement of $\mathrm{He}$ and heavy ion spectra using the IMPACT/SIT, EPACT/STEP and ACE/ULEIS instruments on STEREO, Wind, and ACE, respectively. In addition to differences between the spacecraft expected on the basis of simple corotation, we observed several events where there were markedly different time-intensity profiles from one spacecraft to the next. By comparing the energetic particle intensities and spectral shapes along with solar wind speed we examine the extent to which these differences are due to temporal evolution of the CIR or due to variations in connection to a relatively stable interaction region. Comparing CIRs in the 1996- 1997 solar minimum period vs. 2007-2008, we find that the 2007-2008 period had many more CIRs, reflecting the presence of more high-speed solar wind streams, whereas 1997 had almost no CIR activity.
\end{abstract}

STEREO Science Results at Solar Minimum

Guest Editors: Eric R. Christian, Michael L. Kaiser, Therese A. Kucera, O.C. St. Cyr

G.M. Mason ( $\varangle)$

The Johns Hopkins University Applied Physics Laboratory, Laurel, MD 20723, USA

e-mail: glenn.mason@jhuapl.edu

M.I. Desai

Southwest Research Institute, 6220 Culebra Road, San Antonio, TX 78238, USA

U. Mall · A. Korth · R. Bucik

Max-Planck-Institut für Sonnensystemforschung, Max-Planck-Straße 2, 37191 Katlenburg-Lindau, Germany

T.T. von Rosenvinge

NASA/Goddard Space Flight Center, Greenbelt, MD 20771, USA

K.D. Simunac

Physics Department, Univ. of New Hampshire, Durham, NH 03824, USA 
Keywords Acceleration of particles - Sun: abundances $\cdot$ Solar wind $\cdot$ Interplanetary medium $\cdot$ Cosmic rays $\cdot$ Shock waves

\section{Introduction}

Corotating Interaction Regions (CIRs) are large structures in the interplanetary medium that arise when fast solar wind streams overtake slower solar wind. Plasma turbulence levels increase at the interaction region between the two streams, eventually leading to the formation of shocks beyond $1 \mathrm{AU}$ that move away from the stream interface in both the inward (reverse) and outward (forward) directions. In addition to the plasma interactions, energetic particle intensity increases are often associated with these streams, which were named "corotating" since the intensity increase sometimes appears in repeated solar rotations (for recent reviews on CIRs see Fisk and Jokipii, 1999; Forsyth and Marsch, 1999; Gloeckler, 1999; Gosling and Pizzo, 1999; Horbury and Schmidt, 1999; Mason and Sanderson, 1999; Scholer, 1999; Richardson, 2004).

The common reappearance of CIRs in multiple solar rotations is due to the fact that the underlying coronal structures giving rise to the fast solar wind often evolve slowly; thus to first order CIRs can be considered to be in a nearly steady state. Observations from a single spacecraft sample the CIR only once per solar rotation, so little is known about the evolution on shorter time scales with some exceptions such as a period starting late in 1984 when International Sun Earth Explorer-3 (ISEE-3) and Interplanetary Monitoring Platform-8 (IMP-8) observed CIRs separated by $\sim 1$ day of corotation (Richardson et al., 1993). With the launch of the Solar TErrestrial Relations Observatory (STEREO) spacecraft we have the opportunity to observe CIRs systematically over increasingly large corotation angles, allowing investigation of their behavior over time scales of days, rather than a solar rotation. In this paper we present features of CIR energetic particles during a nearly 2-year period during solar minimum, during which the STEREO spacecraft separation angle reached $80^{\circ}$.

\section{Observations}

The energetic particle observations reported here were obtained with time-of-flight mass spectrometers from the Suprathermal Ion Telescope (SIT, Mason et al., 2008a) instruments on the STEREO-Ahead (-A) and -Behind (-B) spacecraft, the Ultra-Low Energy Isotope Spectrometer (ULEIS, Mason et al., 1998) on the Advanced Composition Explorer (ACE), and the Suprathermal Energetic Particle Telescope (STEP, von Rosenvinge et al., 1995) on the Wind spacecraft. These instruments measure suprathermal heavy ion intensities over the general range $\sim 50 \mathrm{keV}$ nucleon ${ }^{-1}$ to a few $\mathrm{MeV}$ nucleon $^{-1}$. We used the STEREO Plasma and Suprathermal Ion Composition investigation (PLASTIC, Galvin et al., 2008) for solar wind data, along with the Wind and ACE solar wind data (Ogilvie et al., 1995; McComas et al., 1998).

\subsection{Event Selection}

Figure 1 shows an example of STEREO-A observations during late 2007, when the separation angle between STEREO-A and Earth increased from about $16^{\circ}$ to $21^{\circ}$. The three prominent high speed streams reappear for several rotations, although other fast streams such as the one peaking on days 258-260 do not repeat or are much weaker. The CIR intensity increases of suprathermal $\mathrm{He}$ and $\mathrm{O}$ shown in the middle and lower panels are clearly associated with the high speed solar wind streams. The speeds of the suprathermals shown 


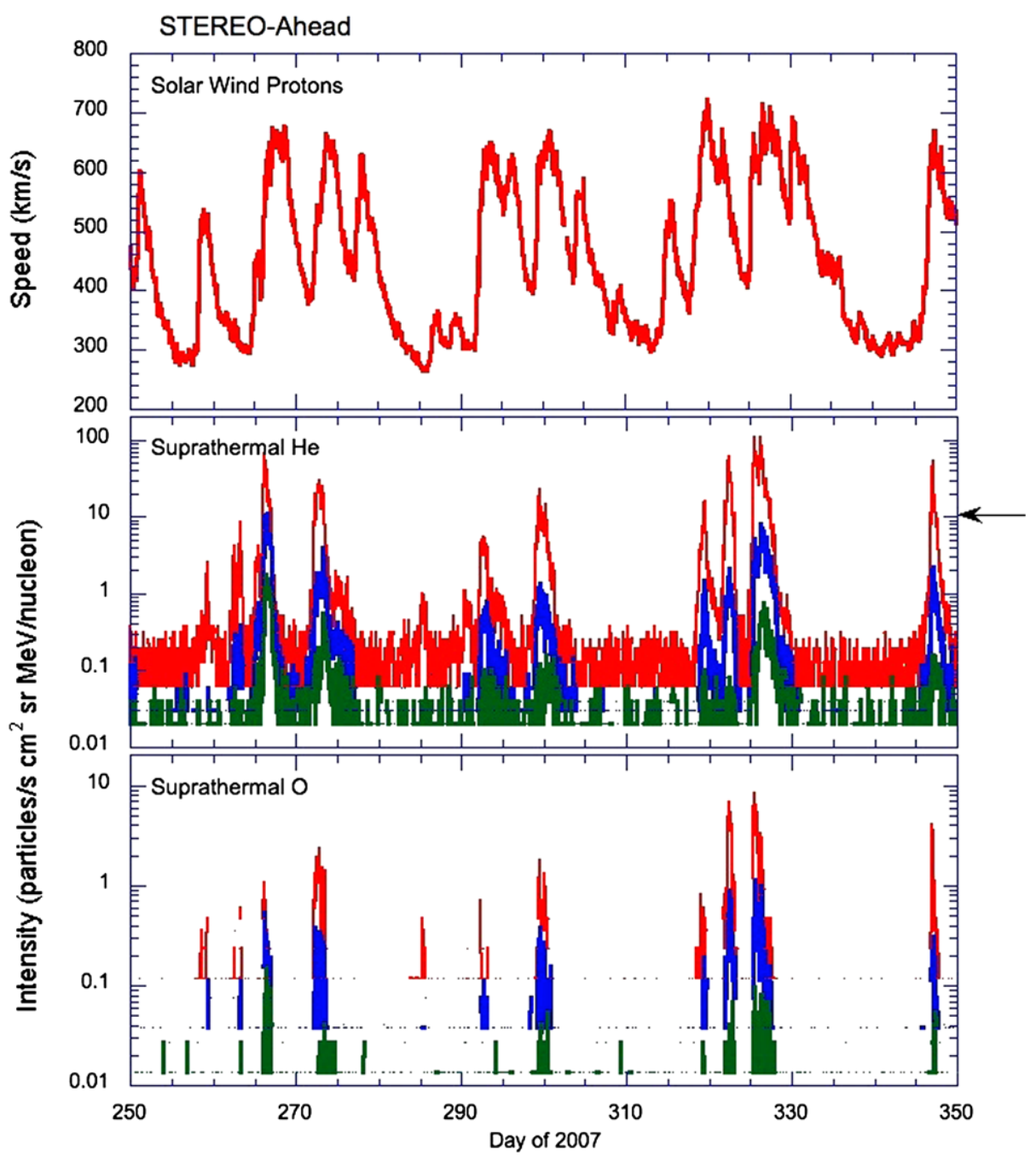

Figure 1 Hourly average values from STEREO-A over a 100 day period in late 2007. Top panel: solar wind proton speed. Middle panel: suprathermal He intensities for 189, 384, and $787 \mathrm{keV}^{\text {nucleon }}{ }^{-1}$. Bottom panel: suprathermal $\mathrm{O}$ intensities for 67,136 , and $266 \mathrm{keV}$ nucleon $^{-1}$. Arrow on middle panel shows approximate selection threshold for CIRs in survey.

are 5-20 times the solar wind speed in the fast streams. Notice that while all the suprathermal intensity increases are associated with high speed streams, there are several streams that do not produce measurable increases in suprathermals, for example those around days 305 , 315 , and 330 .

In order to obtain reasonable statistical accuracy for the suprathermal ion measurements, we required that the hourly average intensity of $189 \mathrm{keV}$ nucleon $^{-1} \mathrm{He}$ exceed 10 particles/( $\left.\mathrm{s} \mathrm{cm}^{2} \mathrm{sr} \mathrm{MeV} \mathrm{nucleon}^{-1}\right)$ on at least one of the two STEREO spacecraft (see arrow on right axis of Figure 1 middle panel) and that the CIR be seen on both STEREO spacecraft and ACE. For example in Figure 1 these criteria excluded energetic particle CIR increases 
Table 1 CIR events

\begin{tabular}{|c|c|c|c|c|c|c|}
\hline $\begin{array}{l}\text { CIR } \\
\#\end{array}$ & $\begin{array}{l}\text { Angle }{ }^{\circ} \\
\text { (a) }\end{array}$ & $\begin{array}{l}\text { SIT-A } \\
\text { Start. . .Stop } \\
\text { Day of year }\end{array}$ & $\begin{array}{l}\text { ULEIS } \\
\text { Start. . Stop } \\
\text { Day of year }\end{array}$ & $\begin{array}{l}\text { SIT-B } \\
\text { Start. . Stop } \\
\text { Day of year }\end{array}$ & $\begin{array}{l}\text { ULEIS peak } \\
\text { intensity (b) }\end{array}$ & $\begin{array}{l}\text { ULEIS spec- Notes } \\
\text { tral index (c) }\end{array}$ \\
\hline
\end{tabular}

\begin{tabular}{|c|c|c|c|c|c|c|c|}
\hline \multicolumn{8}{|c|}{2007} \\
\hline 1 & 0.6 & $29.0 \ldots 31.0$ & $29.5 \ldots 31.5$ & $29.0 \ldots 31.0$ & $5.3 \mathrm{E}+1 \pm 1.9 \mathrm{E}+0$ & $2.56 \pm 0.01$ & $\mathrm{~d}, \mathrm{e}$ \\
\hline 2 & 0.8 & $44.0 \ldots 46.0$ & $44.0 \ldots 46.0$ & $44.0 \ldots 46.0$ & $1.3 \mathrm{E}+1 \pm 5.3 \mathrm{E}-1$ & $2.28 \pm 0.01$ & $\mathrm{~d}, \mathrm{e}$ \\
\hline 3 & 1.2 & $58.5 \ldots 61.0$ & $58.5 \ldots 61.0$ & $58.5 \ldots 61.0$ & $2.0 \mathrm{E}+1 \pm 6.8 \mathrm{E}-1$ & $2.44 \pm 0.01$ & $\mathrm{~d}, \mathrm{e}$ \\
\hline 4 & 1.8 & $72.0 \ldots 75.0$ & $72.0 \ldots 75.0$ & $72.0 \ldots 75.0$ & $3.3 \mathrm{E}+1 \pm 9.1 \mathrm{E}-1$ & $1.72 \pm 0.01$ & $\mathrm{~d}, \mathrm{e}$ \\
\hline 5 & 3.2 & $91.0 \ldots 94.0$ & $91.0 \ldots 94.0$ & $91.0 \ldots 94.0$ & $2.2 \mathrm{E}+0 \pm 2.2 \mathrm{E}-1$ & $3.36 \pm 0.03$ & \\
\hline 6 & 3.9 & $98.5 \ldots 104.0$ & $98.5 \ldots 104.0$ & $98.5 \ldots 104.0$ & $2.1 \mathrm{E}+0 \pm 2.1 \mathrm{E}-1$ & $2.77 \pm 0.02$ & \\
\hline 7 & 7.4 & $127.5 \ldots 133.0$ & $127.5 \ldots 133.0$ & $127.5 \ldots 133.0$ & $5.9 \mathrm{E}+1 \pm 1.8 \mathrm{E}+0$ & $2.46 \pm 0.01$ & $\mathrm{~d}$ \\
\hline 8 & 8.8 & $138.0 \ldots 142.0$ & $138.0 \ldots 142.0$ & $138.0 \ldots 142.0$ & $6.7 \mathrm{E}+0 \pm 3.8 \mathrm{E}-1$ & $2.00 \pm 0.01$ & $\mathrm{~d}$ \\
\hline 9 & 9.7 & $144.0 \ldots 147.0$ & $144.0 \ldots 147.0$ & $144.0 \ldots 147.0$ & $3.4 \mathrm{E}+1 \pm 1.4 \mathrm{E}+0$ & $2.77 \pm 0.01$ & \\
\hline 10 & 11.5 & $154.0 \ldots 159.0$ & $154.0 \ldots 159.0$ & $154.0 \ldots 159.0$ & $8.7 \mathrm{E}+0 \pm 4.4 \mathrm{E}-1$ & $3.20 \pm 0.02$ & \\
\hline 11 & 18.6 & $192.0 \ldots 195.0$ & $192.0 \ldots 195.0$ & $191.5 \ldots 195.5$ & $5.2 \mathrm{E}+1 \pm 2.1 \mathrm{E}+0$ & $3.07 \pm 0.01$ & \\
\hline 12 & 20.7 & $202.0 \ldots 206.0$ & $202.0 \ldots 206.0$ & $201.0 \ldots 205.0$ & $3.8 \mathrm{E}-1 \pm 9.0 \mathrm{E}-2$ & $3.73 \pm 0.08$ & $\mathrm{f}$ \\
\hline 13 & 24.0 & $219.0 \ldots 222.0$ & $219.0 \ldots 222.0$ & $218.0 \ldots 221.0$ & $2.1 \mathrm{E}+0 \pm 2.1 \mathrm{E}-1$ & $2.54 \pm 0.02$ & \\
\hline 14 & 28.3 & $239.0 \ldots 247.0$ & $238.0 \ldots 246.0$ & $237.5 \ldots 246.0$ & $2.1 \mathrm{E}+0 \pm 2.2 \mathrm{E}-1$ & $1.44 \pm 0.03$ & \\
\hline 15 & 32.5 & $264.0 \ldots 268.0$ & $264.0 \ldots 267.0$ & $262.0 \ldots 266.0$ & $1.1 \mathrm{E}+1 \pm 4.8 \mathrm{E}-1$ & $2.30 \pm 0.02$ & \\
\hline 16 & 33.7 & $271.5 \ldots 274.0$ & $270.5 \ldots 274.0$ & $269.5 \ldots 272.0$ & $2.6 \mathrm{E}+0 \pm 2.4 \mathrm{E}-1$ & $2.08 \pm 0.02$ & \\
\hline 17 & 37.6 & $298.5 \ldots 302.0$ & $298.0 \ldots 301.0$ & $297.5 \ldots 300.0$ & $6.8 \mathrm{E}+0 \pm 4.1 \mathrm{E}-1$ & $3.62 \pm 0.02$ & \\
\hline 18 & 39.9 & $318.0 \ldots 321.0$ & $317.0 \ldots 321.0$ & $316.5 \ldots 319.0$ & $8.5 \mathrm{E}-1 \pm 1.3 \mathrm{E}-1$ & $3.86 \pm 0.06$ & \\
\hline 19 & 40.2 & $321.5 \ldots 323.5$ & $321.5 \ldots 323.5$ & $320.5 \ldots 324.0$ & $6.4 \mathrm{E}-2 \pm 3.7 \mathrm{E}-2$ & $3.76 \pm 0.34$ & \\
\hline 20 & 40.5 & $325.0 \ldots 328.0$ & $324.0 \ldots 327.0$ & $324.0 \ldots 328.0$ & $5.2 \mathrm{E}+1 \pm 2.4 \mathrm{E}+0$ & $3.30 \pm 0.01$ & \\
\hline 21 & 42.1 & $346.0 \ldots 349.0$ & $344.0 \ldots 347.0$ & $343.0 \ldots 346.0$ & $4.0 \mathrm{E}+0 \pm 2.9 \mathrm{E}-1$ & $3.05 \pm 0.02$ & \\
\hline 22 & 42.6 & $353.0 \ldots 356.0$ & $351.0 \ldots 355.0$ & $350.0 \ldots 354.0$ & $4.3 \mathrm{E}+1 \pm 1.8 \mathrm{E}+0$ & $2.97 \pm 0.01$ & \\
\hline \multicolumn{8}{|c|}{2008} \\
\hline 23 & 43.7 & $6.0 \ldots 15.0$ & $4.5 \ldots 15.0$ & $3.5 \ldots 13.0$ & $1.3 \mathrm{E}+1 \pm 5.3 \mathrm{E}-1$ & $1.88 \pm 0.01$ & \\
\hline 24 & 44.6 & $33.0 \ldots 36.0$ & $31.5 \ldots 35.0$ & $29.5 \ldots 33.5$ & $3.7 \mathrm{E}+0 \pm 2.8 \mathrm{E}-1$ & $1.81 \pm 0.02$ & \\
\hline 25 & 45.0 & $42.5 \ldots 45.0$ & $41.0 \ldots 45.0$ & $40.0 \ldots 43.0$ & $6.6 \mathrm{E}+1 \pm 2.1 \mathrm{E}+0$ & $2.46 \pm 0.01$ & \\
\hline 26 & 45.7 & $61.0 \ldots 63.0$ & $58.0 \ldots 62.0$ & $57.5 \ldots 60.0$ & $7.7 \mathrm{E}+0 \pm 4.1 \mathrm{E}-1$ & $1.90 \pm 0.02$ & \\
\hline 27 & 46.1 & $69.5 \ldots 73.0$ & $69.0 \ldots 71.0$ & $67.0 \ldots 70.0$ & $2.4 \mathrm{E}+0 \pm 2.3 \mathrm{E}-1$ & $3.05 \pm 0.04$ & \\
\hline 28 & 47.2 & $88.0 \ldots 91.5$ & $86.0 \ldots 90.0$ & $85.0 \ldots 87.0$ & $5.4 \mathrm{E}+0 \pm 3.5 \mathrm{E}-1$ & $1.99 \pm 0.02$ & \\
\hline 29 & 48.0 & $98.0 \ldots 101.0$ & $96.0 \ldots 99.5$ & $94.0 \ldots 97.0$ & $1.5 \mathrm{E}+0 \pm 1.8 \mathrm{E}-1$ & $2.85 \pm 0.04$ & \\
\hline 30 & 49.5 & $115.5 \ldots 117.4$ & $113.5 \ldots 115.5$ & $112.0 \ldots 114.5$ & $6.2 \mathrm{E}-1 \pm 1.2 \mathrm{E}-1$ & $3.84 \pm 0.08$ & $\mathrm{~g}$ \\
\hline 31 & 57.1 & $168.5 \ldots 171.0$ & $167.0 \ldots 171.0$ & $165.5 \ldots 169.0$ & $7.2 \mathrm{E}-1 \pm 1.2 \mathrm{E}-1$ & $1.89 \pm 0.05$ & \\
\hline 32 & 67.2 & $224.0 \ldots 228.0$ & $222.0 \ldots 226.0$ & $220.0 \ldots 224.0$ & $4.9 \mathrm{E}+1 \pm 2.1 \mathrm{E}+0$ & $3.43 \pm 0.01$ & \\
\hline 33 & 69.1 & $234.0 \ldots 238.0$ & $231.0 \ldots 237.0$ & $229.0 \ldots 232.0$ & $2.4 \mathrm{E}-1 \pm 7.1 \mathrm{E}-2$ & $3.45 \pm 0.08$ & $\mathrm{~h}$ \\
\hline 34 & 71.9 & $250.5 \ldots 254.0$ & $247.5 \ldots 250.5$ & $245.5 \ldots 249.0$ & $4.9 \mathrm{E}-1 \pm 1.0 \mathrm{E}-1$ & $2.82 \pm 0.07$ & \\
\hline 35 & 76.7 & $277.5 \ldots 282.0$ & $275.0 \ldots 280.0$ & $272.0 \ldots 277.0$ & $1.1 \mathrm{E}+1 \pm 4.9 \mathrm{E}-1$ & $1.76 \pm 0.01$ & \\
\hline 36 & 80.6 & $305.5 \ldots 309.0$ & $302.5 \ldots 305.0$ & $299.0 \ldots 305.0$ & $1.4 \mathrm{E}+0 \pm 1.7 \mathrm{E}-1$ & $2.49 \pm 0.04$ & \\
\hline
\end{tabular}

Notes:
(a) STEREO-B to Sun to STEREO-A angle at CIR mid-point at ACE.
(b) Particles/( $\mathrm{s} \mathrm{cm}^{2} \mathrm{sr} \mathrm{MeV}$ nucleon $^{-1}$ ) over energy $0.32-0.45 \mathrm{MeV}$ nucleon $^{-1}$ (1-hr averaged).
(c) Assuming power law $\mathrm{d} J / \mathrm{d} E=E^{-\gamma}$ over range $0.16-0.91 \mathrm{MeV}$ nucleon $^{-1}$ (event averaged).
(d) Event in survey of Mason et al. (2008b).
(e) Event in survey of Leske et al. (2008).
(f) Very small event on SIT-A.
(g) Insufficient intensity on SIT-B for spectral fit.
(h) Event very small on ULEIS and SIT-A. 
Figure 2 Day of Bartels rotation for each CIR in Table 1 vs. the time of the CIR at ACE (year).

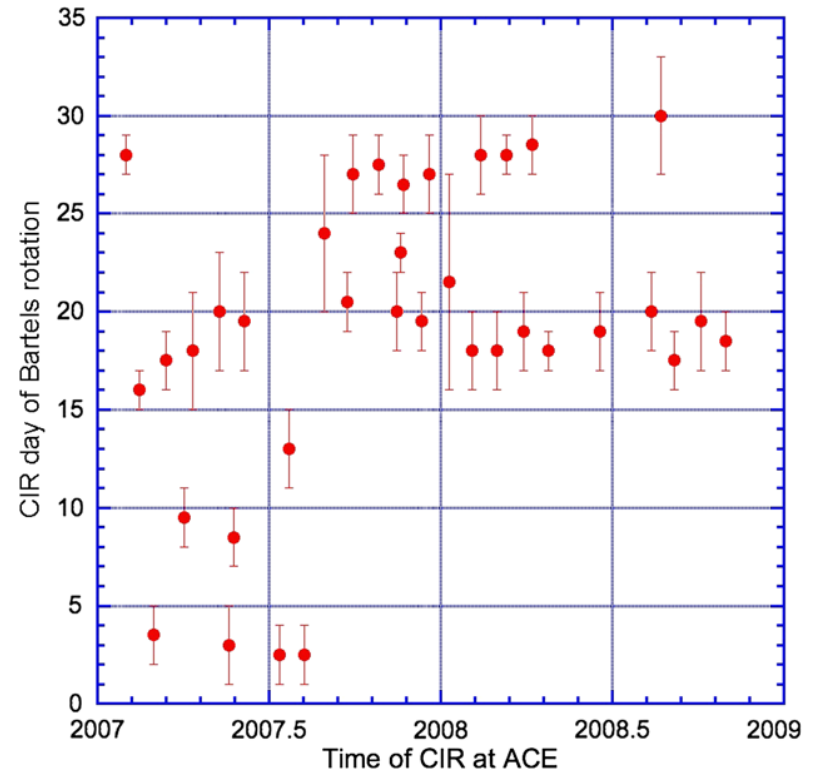

on days 258, 262, 284, and 291. Table 1 lists 36 CIRs identified between late January 2007 (when both SIT instruments became operational) and early November 2008. Column 1 is the event number and column 2 is the STEREO-B to Sun to STEREO-A angle at approximately the midpoint of the time interval of the CIR observed at ULEIS. Columns 3, 4, and 5 list the approximate start and end times at each of the spacecraft; since the CIRs often show slow intensity increases, the start and end times are nominal only. Column 6 lists the ULEIS peak hourly average intensity for $0.32-0.45 \mathrm{MeV}^{\text {nucleon }}{ }^{-1} \mathrm{He}$ during the CIR interval. Column 7 contains the spectral index from fits to the $0.16-0.91 \mathrm{MeV}$ nucleon ${ }^{-1} \mathrm{He}$ spectrum, assuming a differential power law in kinetic energy per nucleon. Column 8 has notes on several of the CIRs.

Figure 2 shows the time of each CIR in Table 1 during Bartels solar rotations vs. time of the CIR at ACE. Recurring events appear in the figure as a series with nearly the same day of Bartels rotation. Although there were several sources in 2007, by 2008 a fairly stable pattern emerged with two regions being responsible for all the cases in the table.

\subsection{Spectral Form}

Figure 3 shows particle spectra for He, C, O, and Fe for the 10 February 2008 CIR, one of the most intense in the survey. The spectral forms show low energy power laws of similar shape for different species; the spectra steepen significantly above $\sim 1 \mathrm{MeV}$ nucleon ${ }^{-1}$. The spectral shape and composition are typical of CIRs (Gloeckler, Hovestadt, and Fisk, 1979; Mason et al., 1997, 2008b; Desai et al., 1998; Richardson, 2004). The dashed line in the figure shows the He spectral shape from the 22 March 2000 CIR, an intense event included in the 1998-2007 survey on ACE (Mason et al., 2008b). Near $100 \mathrm{keV}$ nucleon $^{-1}$ the $10 \mathrm{Feb}-$ ruary CIR intensity is close to the 22 March intensity; however, above $1 \mathrm{MeV}$ nucleon $^{-1}$ the 10 February CIR spectrum becomes much steeper so that by energies of $\sim 3 \mathrm{MeV}$ nucleon $^{-1}$ its intensity is nearly a factor of 50 lower.

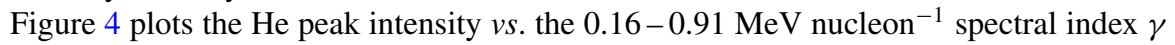
assuming a power law of the form $\mathrm{d} J / \mathrm{d} E \propto E^{-\gamma}$, where $E$ is kinetic energy per nucleon. In 
Figure 3 ACE/ULEIS spectra for the CIR starting 10 February 2008 (\#25 in Table 1). Filled red circles: He, inverted diamonds: $\mathrm{C}$, lower filled squares: O, upper filled squares: Fe, dashed line: He spectral shape measured in the 22 March 2000 CIR (Mason et al., 2008b).
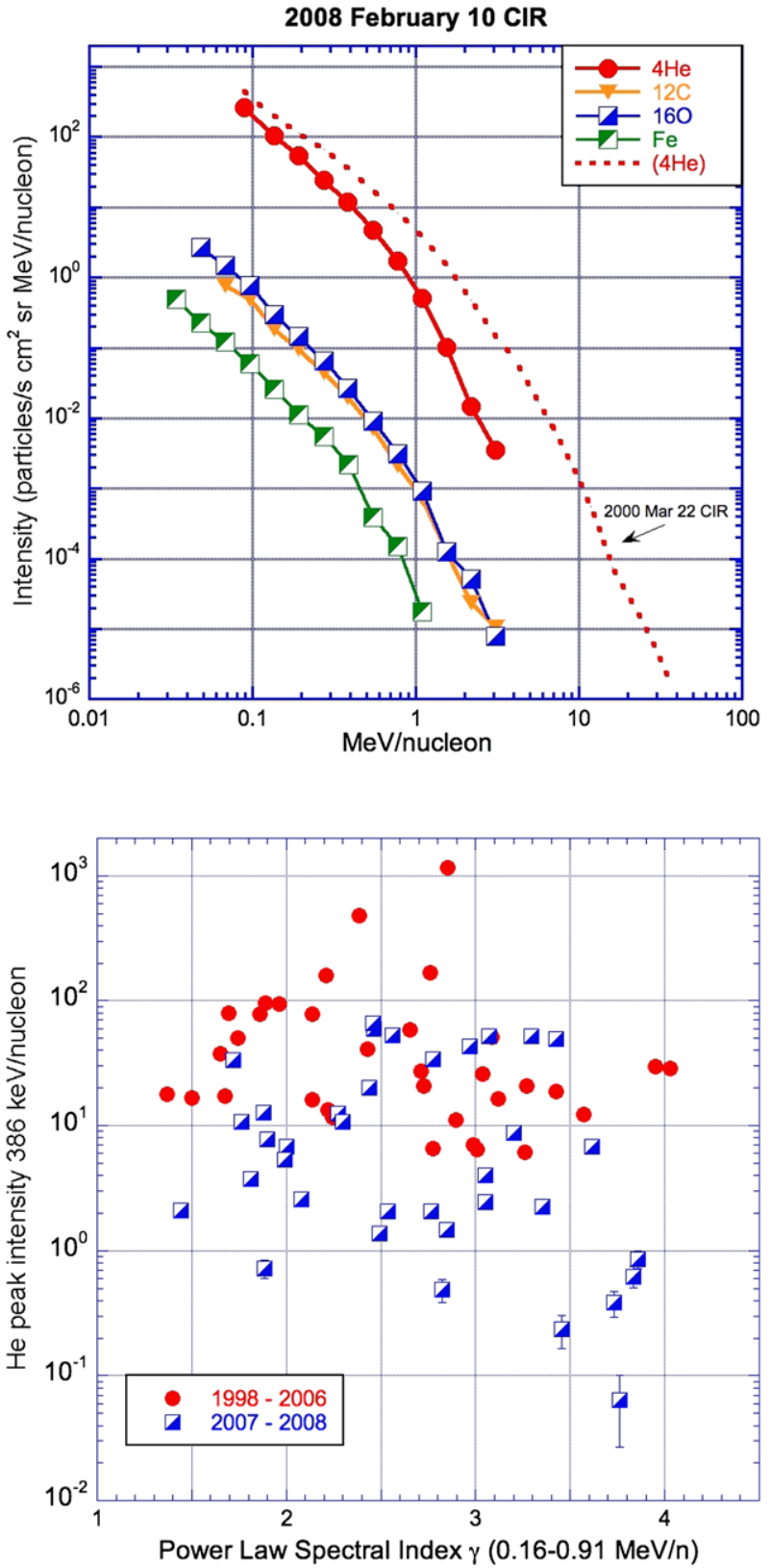

Power Law Spectral Index $\gamma(0.16-0.91 \mathrm{MeV} / \mathrm{n})$
Figure 4 Peak intensity for $386 \mathrm{keV}$ nucleon $^{-1} \mathrm{He} v s$. differential energy power law spectral index over the range 0.16-0.91 MeV nucleon ${ }^{-1}$ for 1998 - 2006 (filled red circles), and $2007-2008$ (half-filled squares, values in Table 1).

the figure, events from the 1998-2006 period are compared with the current set of 20072008 CIRs. It is clear that there is no strong correlation between spectral index and peak intensity - this may indicate that connection to the CIR, and not the CIR shock properties themselves, are the primary factor in determining the intensity. The mean values of the spectral indices are $2.57 \pm 0.12$ for the $1998-2006$ events and $2.69 \pm 0.11$ for $2007-2008$; although the $2007-2008$ spectra are somewhat steeper, the difference is only $\sim 1$-sigma. 
Figure 5 Geocentric Solar Ecliptic positions of STEREO-Ahead and STEREO-Behind from launch through 2008 day 343. Numbers by each position trace indicate day of 2007 or 2008 . The green Archimedes spiral line is for a nominal $650 \mathrm{~km} \mathrm{~s}^{-1}$ solar wind speed.

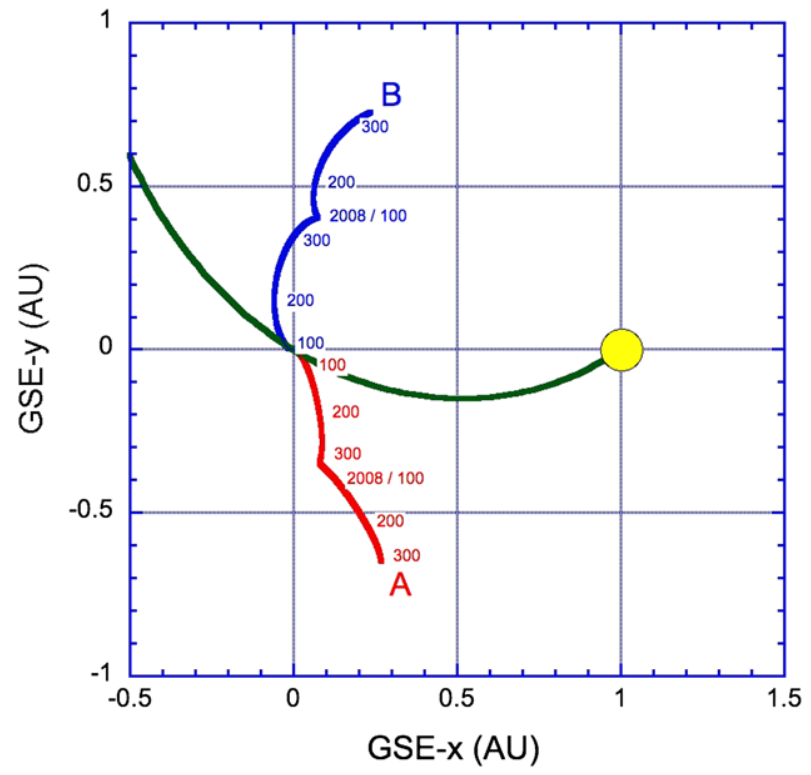

\subsection{Corotation features}

Figure 5 shows the overall trajectories of the STEREO spacecraft during 2007-2008. Up to roughly 2007 day 100 the two spacecraft were close to being on the same nominal Archimedes spiral field line. Therefore for the first few CIRs in Table 1 both STEREO spacecraft were close enough together to allow a cross-calibration with each other and with ACE instruments. Not until the second half of 2007 were the two STEREO spacecraft separated by more than one day of corotation, and so the CIR signatures seen on both were similar, but not identical (see Leske et al., 2008). As the STEREO spacecraft moved further apart, the Behind spacecraft CIR events "led" the Ahead spacecraft by increasing amounts. Figure 6 shows spectrograms of SIT-A and SIT-B CIRs for the month of August 2008, when the angular separation between the two spacecraft corresponded to about five days of corotation. The figure shows that while some features are clearly seen by both SIT-B and SIT-A, this is not always the case. For example, the intense event starting around day 220 on SIT-B is clearly seen on SIT-A around day 225 . However, the CIR related brief intensity increases on days 224-227 seen by SIT-B have no clear signature at SIT-A. The intense CIR starting on day 229 at SIT-B is seen only faintly on SIT-A. Finally, we see that a faint increase at SIT-A starting around day 240 had no obvious predecessor at SIT-B.

Figure 7 examines these properties by plotting a single time-intensity profile over the same set of events seen in the spectrogram in Figure 6. The panels in Figure 7 have been offset by the nominal rotation time so that rigidly corotating features would line up. Note that the event onset at day 220 on STEREO-B has its onset at ACE at almost precisely the time expected from corotation; however, at STEREO-A the onset is almost a full day earlier than expected from corotation. Notice also that the sharp, intense increases seen on STEREO-B on days 224-226 are not detected by ACE or STEREO-A. The strong increase starting on day 230 on STEREO-B has a markedly different appearance on ACE and STEREO-A.

While the CIRs we observe are doubtless undergoing temporal changes, it is also the case that CIRs often last for many solar rotations (Figure 2) so the large differences shown 


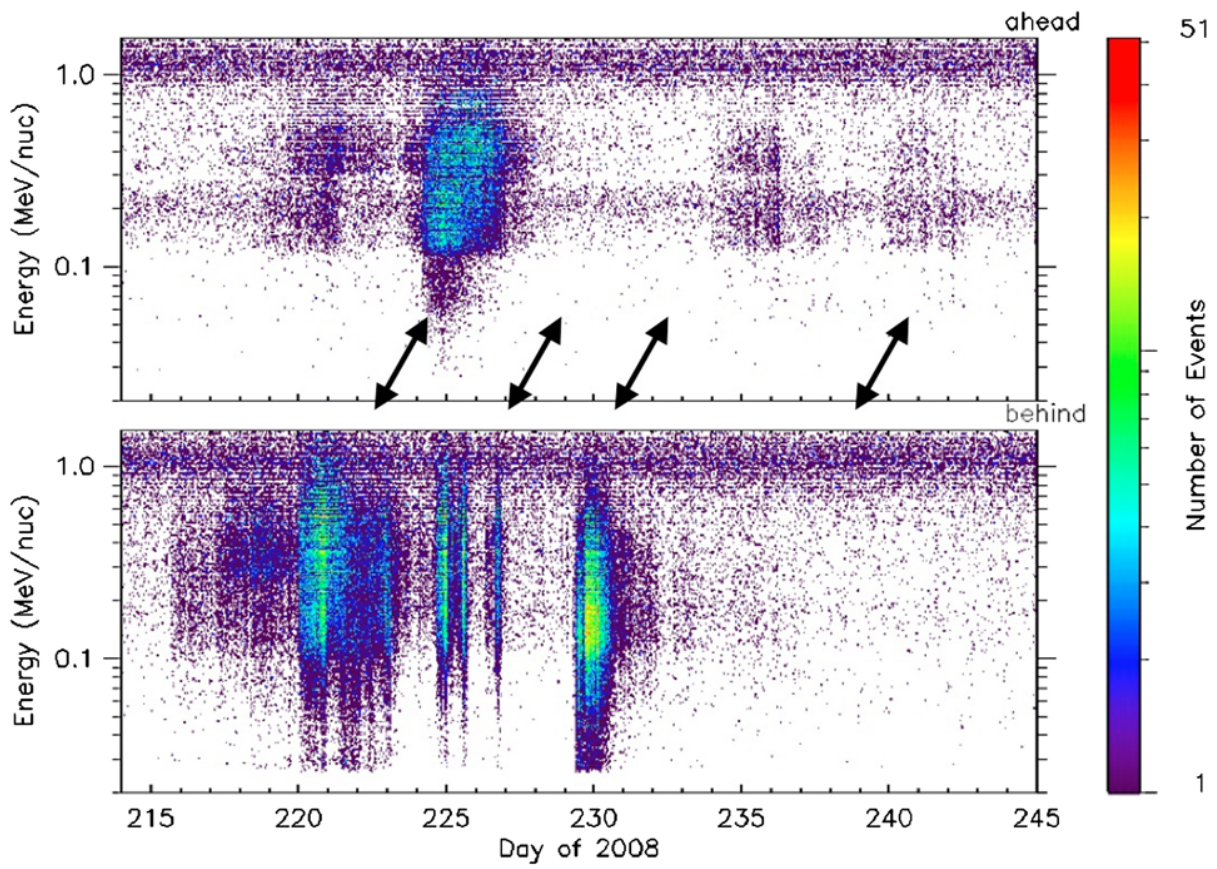

Figure 6 Spectrograms of ion energy $v s$. arrival time for SIT-A (upper panel) and SIT-B (lower panel) for August 2008. During this period the angular separation of the two STEREO spacecraft increased from $65.4^{\circ}$ to $71.2^{\circ}$, or roughly five days of corotation. Double ended arrows point to nominally associated features, or features that were observed on one spacecraft by not the other.

in Figure 7 over periods of $\sim 0.1$ solar rotations must generally be dominated by effects other than temporal evolution of the CIR. Figure 8 shows the $19.5 \mathrm{~nm}$ solar image on 2008 day 220, where the coronal hole giving rise to event \#32 can be clearly seen with location $20-40^{\circ}$ west of the central meridian (CM) seen from STEREO-B. Since $650 \mathrm{~km} \mathrm{~s}^{-1}$ solar wind takes about 2.5 days to reach $1 \mathrm{AU}$, the coronal hole at $20-40^{\circ}$ west is at the expected location as the source of event \#32. The shape of the hole is irregular and it is qualitatively reasonable that small differences in spacecraft tracks across the hole could lead to different remote signatures. The double headed arrow in the figure shows the size of the latitude difference between STEREO-B and -A at this time $\left(5.2^{\circ}\right)$, which is comparable to the scale size of features in the coronal hole. The coronal hole seen below the equator at east $\sim 30-60^{\circ}$ apparently corresponds to the short intense events seen on STEREO-B four days after the passage of event \#32. The fact that this coronal hole is below the equator may explain why STEREO-A and ACE (both with subsolar points north of the equator) did not see this event. Leske et al. (2008) have discussed the importance of latitude effects in interpretation of STEREO CIRs and Sanderson et al. (1998) have shown the importance of these effects on data from the Wind spacecraft.

While the intensities and their temporal evolution sometimes show the significant differences illustrated in Figures 6 and 7, the spectral indices observed by the different spacecraft show much less variation. In the generally accepted view of CIR formation, the spectral index in a CIR is determined by the acceleration properties at the forward- and reverse- shocks (e.g., Fisk and Lee, 1980) and this might be expected to be a less variable feature than details of the connection to the distant shock that could affect the hourly intensities. Figure 9 shows 
Figure 7 Hourly average $189 \mathrm{keV}$ nucleon ${ }^{-1} \mathrm{He}$ intensities from SIT-A (red, top panel), ACE/ULEIS (green, middle panel), and SIT-B (blue, bottom panel). The time axes of the plots have been shifted by the nominal corotation delays such that steady corotating features would line up on the page. The dashed line is to guide the eye for the onset of the CIR starting 2008 day 222 at ACE (event \#32 in Table 1).

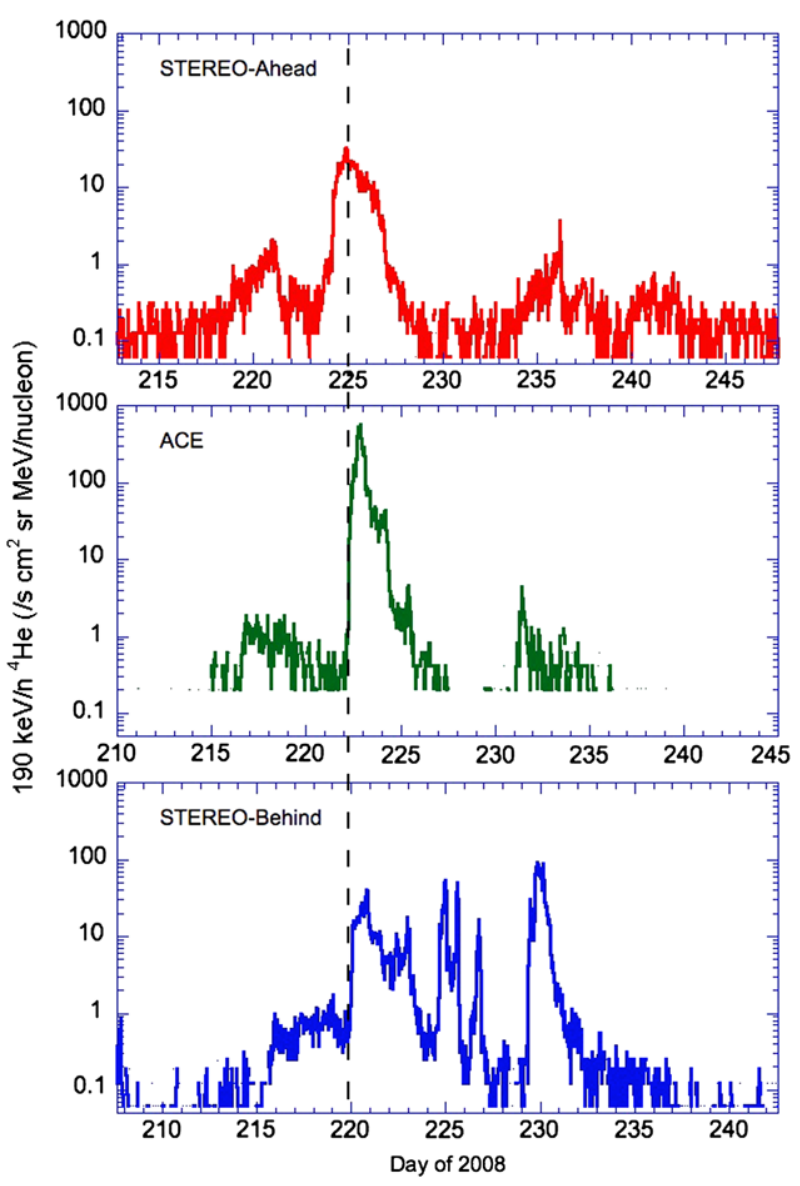

the difference in the $0.16-0.91 \mathrm{MeV}$ nucleon $^{-1} \mathrm{He}$ spectral index measured on SIT-A and SIT-B, plotted vs. the heliographic latitude difference between the two spacecraft. Note that many of the cases have spectra very close to each other: their spectral indices differ by less than 0.5. As the latitude difference gets larger, the spectral differences increase, leading to a positive correlation of 0.61 , which is highly significant for a sample of 35 cases (CIR \#30 did not have a measurable He spectrum for SIT-B). Thus, the spectra are generally in close agreement between SIT-B and SIT-A and the differences increase as the spacecraft heliolatitude differences increase.

\subsection{Solar Cycle Effects}

Figure 10 shows $0.32-0.45 \mathrm{MeV}$ nucleon $^{-1}$ peak hourly He intensities for $84 \mathrm{CIRs}$ from late 1994 through the present. The Wind/STEP points (red diamonds) have been corrected to take account of the differences between the on-board rate energy bands for STEP $v s$. ACE/ULEIS. The largest events occur during solar active periods. We examined the four most intense events to see if they had some other characteristics that could help to explain this. Two of the events (27 July 2003 and 28 January 2000) were associated with large coronal hole structures rather than the more modest scale coronal holes typical of the CIRs in 
Figure 8 Sun Earth Connection Coronal and Heliospheric Investigation-B (SECCHI-B) $19.5 \mathrm{~nm}$ image taken on 2008 day 220 showing the coronal hole feature giving rise to the event \#32 shown in Figure 7. Blue longitude line is central meridian (CM) seen from STEREO-B, green is $\mathrm{CM}$ seen from $\mathrm{ACE}$, red is CM seen from STEREO-A. Double headed arrow near CM shows the approximate latitude difference between STEREO-A and $-\mathrm{B}$ at this time. Grid on image has $10^{\circ}$ spacing.

Figure 9 Difference between SIT-A and SIT-B spectral index for He vs. heliographic latitude difference between the two spacecraft. The correlation coefficient between the two quantities is 0.61 , which has a $<0.1 \%$ chance of arising from unrelated variables.
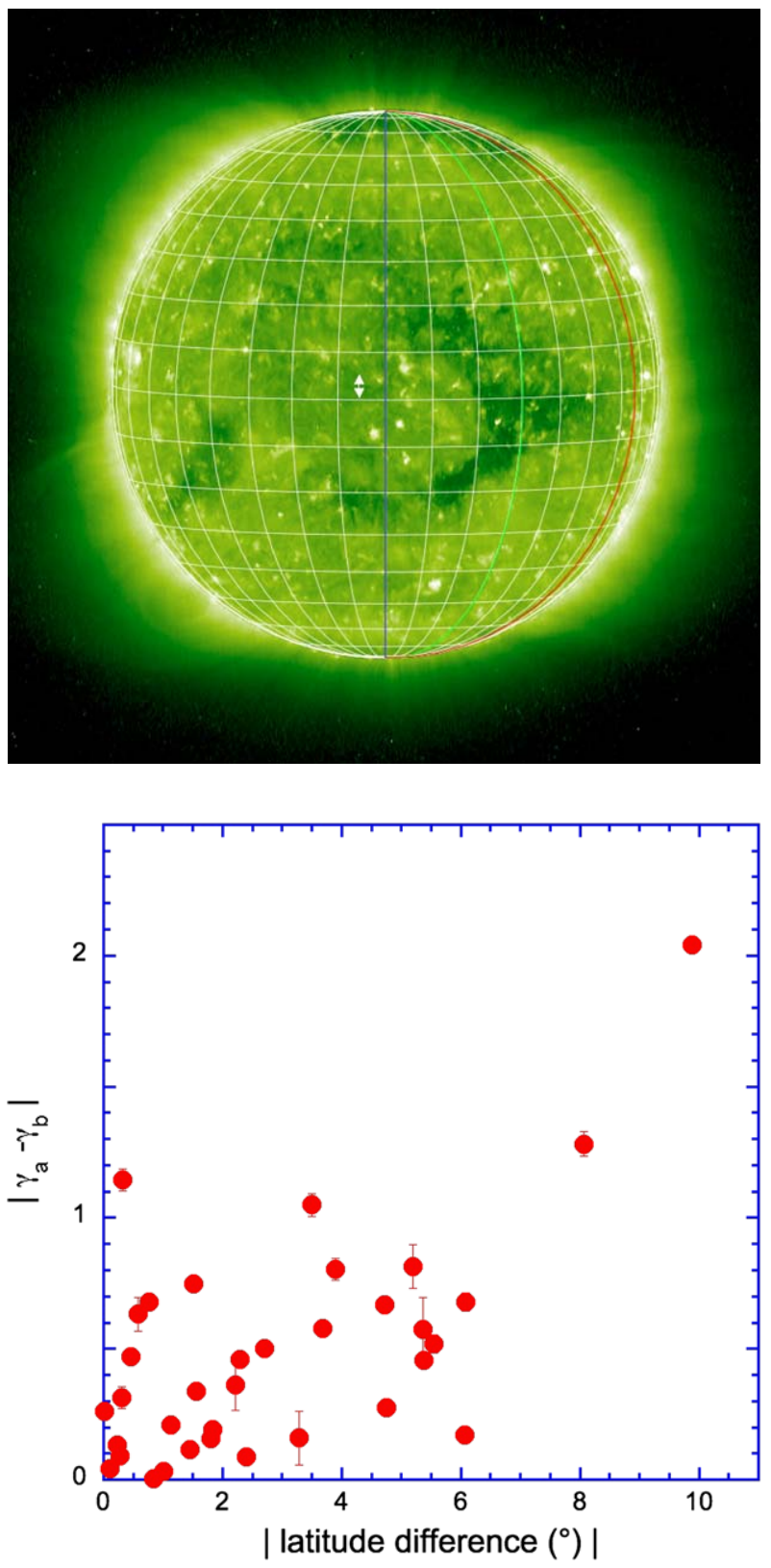

the present survey. However, the other two events (15 November 2003 and 8 October 2005) were associated with coronal holes not obviously different from the one shown in Figure 8.

The average solar wind speed for these four most intense events was $763 \pm 37 \mathrm{~km} \mathrm{~s}^{-1}$, 1-2 sigma higher than the whole set in the survey of Mason et al. (2008b); however, in that set of 41 CIRs, there were several other events with high peak solar wind speeds whose peak suprathermal ion intensities were lower than the four most intense CIR events. These four most intense CIR events were associated with forward or reverse shocks, which are 
Figure 10 CIR peak hourly intensities of $386 \mathrm{keV}$ nucleon $^{-1}$ He from Wind/STEP (red diamonds) ACE/ULEIS 1998 - 2006 (blue filled circles) and ACE/ULEIS current 2007-2008 survey (green half-filled squares). The orange line corresponds to the monthly smoothed sunspot number (right axis). $1994-1997$ points are from Mason et al. (1997), with two additional events added for this plot and 1998-2006 data from Mason et al. (2008b).

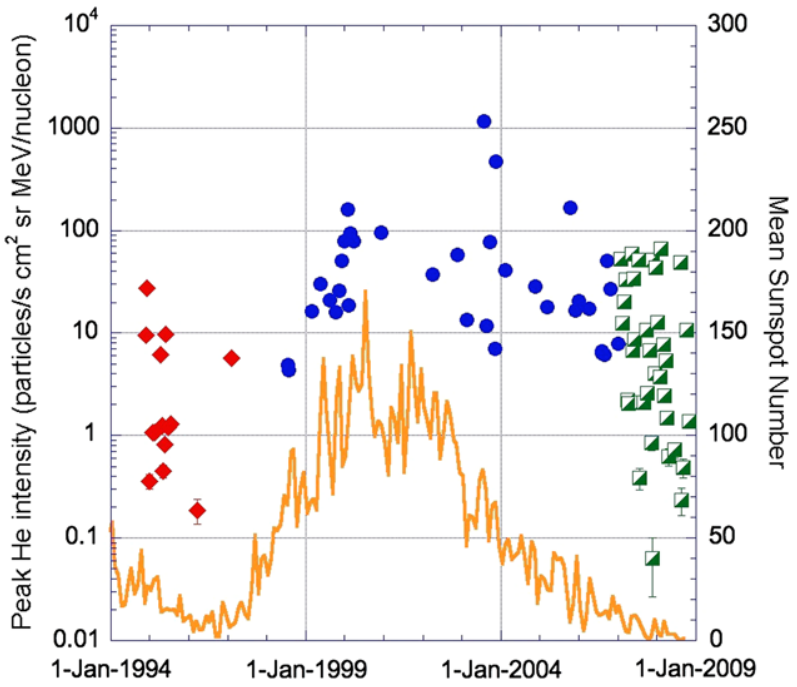

somewhat unusual for 1 AU CIRs. For example, in the survey of Jian et al. (2006) only about 50\% of the ACE survey CIRs (Mason et al., 2008b) from 1998-2004 had associated shocks at $1 \mathrm{AU}$. It has been long known that at the suprathermal particle energies studied here, it is difficult for the ions to propagate in from distant shocks (Fisk and Lee, 1980; Mason et al., 1999) and so local acceleration by compression regions may play a critical role (Giacalone, Jokipii, and Kóta, 2002). The presence of the 1 AU shocks could be an indicator for local acceleration; however, the shock jumps for these four most intense CIRs are not consistently stronger than for other less intense events that also showed shocks.

Another possibility is that the ambient suprathermal ion density was enhanced in these CIRs, resulting in a higher maximum intensity; while all four events indeed had elevated suprathermal ion densities beforehand, many other events in the survey had similarly elevated intensities without producing intense CIRs. Solar wind speed, coronal hole size, the presence of forward or reverse shocks at $1 \mathrm{AU}$, and elevated ambient intensities could be all expected to produce more intense events, which while consistent with these associations, does not explain the peak intensities in any simple way. See Simnett et al. (1994) and Gopalswamy et al. (2002) for discussions of the effects of ambient populations on CIRs and interplanetary shock accelerated particles.

With the exception of the four CIRs discussed above, all the other CIRs shown in Figure 10 have maximum intensities below 100 particles $/\left(\mathrm{s} \mathrm{cm}^{2} \mathrm{sr} \mathrm{MeV} \mathrm{nucleon}^{-1}\right)$. The distribution of CIR peak intensities at $386 \mathrm{keV}$ nucleon ${ }^{-1}$ has a much sharper cutoff than is seen in solar energetic particles (SEPs) at higher energy, as illustrated in Figure 11, which shows the distribution for the entire period (red shading) and also the 2007-2008 solar minimum subset (blue hatching). The SEP distribution has a slope of about -0.4 , as reported previously by Reedy (1996, quoted by McCracken et al., 2001), although we note that Van Hollebeke, Ma Sung, and McDonald (1975) obtained a slope of $-1.15 \pm 0.1$ with a different selection of events. Below peak intensities of 10 particles $/\left(\mathrm{s} \mathrm{cm}^{2} \mathrm{sr} \mathrm{MeV} \mathrm{nucleon}{ }^{-1}\right)$, the CIR 1994-2008 distribution is flattened artificially due to a masking of small CIRs during solar active periods such that no small CIRs are in the 1998-2006 period. But for the higher intensities during 1994-2008, and also for the 2007-2008 period this is not a problem, and looking at the right panel of Figure 11 it is evident that at the low energies of this survey there is a fairly sharp limit on CIR intensity. In particular the 2007-2008 period shows a flat 

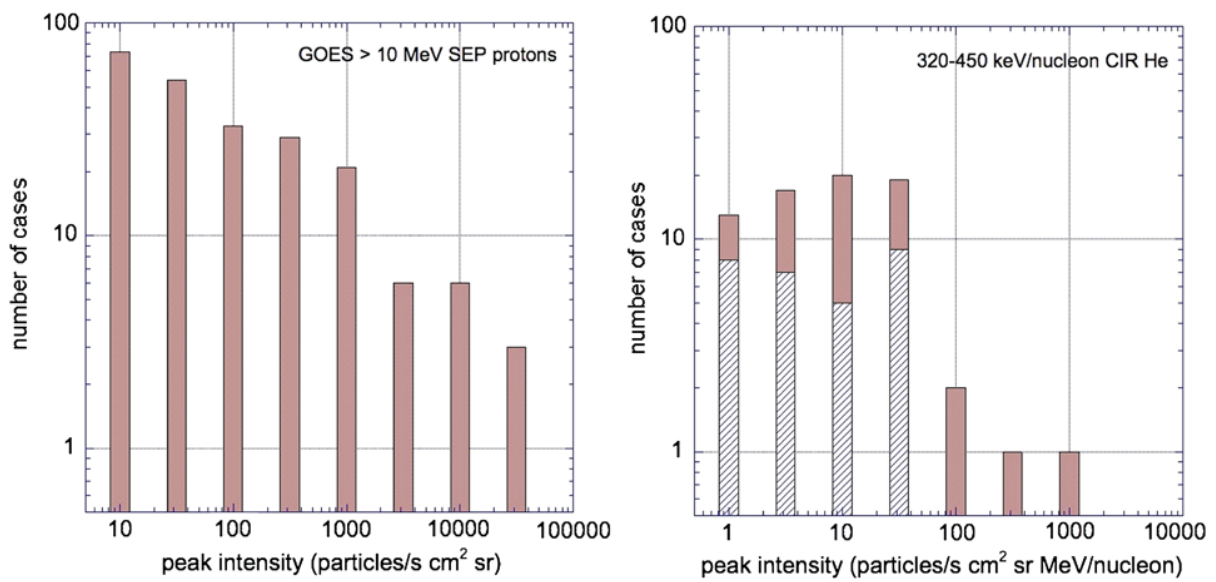

Figure 11 Left: histogram of peak event intensities for $10 \mathrm{MeV}$ protons for 225 solar energetic particle events observed by GOES satellites from 1976-2006 (from NOAA web site: http://www.swpc.noaa.gov/ ftpdir/indices/SPE.txt). Right: peak event intensities for $320-450 \mathrm{keV}$ nucleon ${ }^{-1} \mathrm{He}$ for CIR events observed from 1994-2008 (red shading) and 2007-2008 survey subset (blue hatching). Below peak intensities of $\sim 10$ particles/( $\mathrm{s} \mathrm{cm}^{2} \mathrm{sr} \mathrm{MeV}$ nucleon $\left.^{-1}\right)$ the CIR histogram is missing events during solar active periods due to SEP background, but this does not affect the 2007-2008 subset.

number distribution $v s$. peak intensity with a sharp cutoff. This may be in part due to the fact that the CIR high speed solar wind streams do not show a large variation of speed, but given other factors involved such as coronal hole size and duration, and details of connection to the CIR beyond $1 \mathrm{AU}$, the relative sharpness of the cutoff seems surprising.

\subsection{Comparison of 1996-1997 vs. 2007 -2008 Solar Minimum Periods}

Figure 12 compares solar wind and suprathermal particle intensities for the 1996-1997 vs. 2007-2008 periods near solar minimum. The energy of suprathermal He detected by STEP from the Energetic Particles: Acceleration, Composition and Transport (EPACT) investigation is $\sim 386 \mathrm{keV}$ nucleon ${ }^{-1}$, adjusted as in Figure 10 to align the STEP energy band with ACE/ULEIS. In the figure, solar wind data taken closer than 25 Earth radii have been blanked out to remove magnetospheric effects. Suprathermals have been blanked out during the November 1997 SEP events and during a large interplanetary shock on 1997 day 326. The differences between the two solar minima are quite evident in the figure; although there were regular episodes of high- and low-speed solar wind streams in both minima, the pattern in 2007-2008 is much more regular. The peak speed of the high speed streams is much lower in 1996-1997 than during 2007-2008 and shows an overall decrease from 1996 to 1997 that is not evident in the 2007-2008 data. Similar properties are seen in the suprathermal He intensities, during 1996-1997 the peak intensities are a factor of 5-10 lower than during 2007-2008 and the number of events is smaller. There is some variation also in the 2007-2008 data with 2008 being relatively less active than 2007, but nevertheless there were intense CIRs occurring in late 2008.

\section{6. "Dropouts" During CIRs}

Several CIRs in the survey (numbers 2, 6, 7, 10, 14, 16, 33 in Table 1) show episodes on one or more of the spacecraft of energy-independent intensity decreases by a factor of 5-10 followed by an increase a few hours later. Figure 13 shows an example of such an event, where 


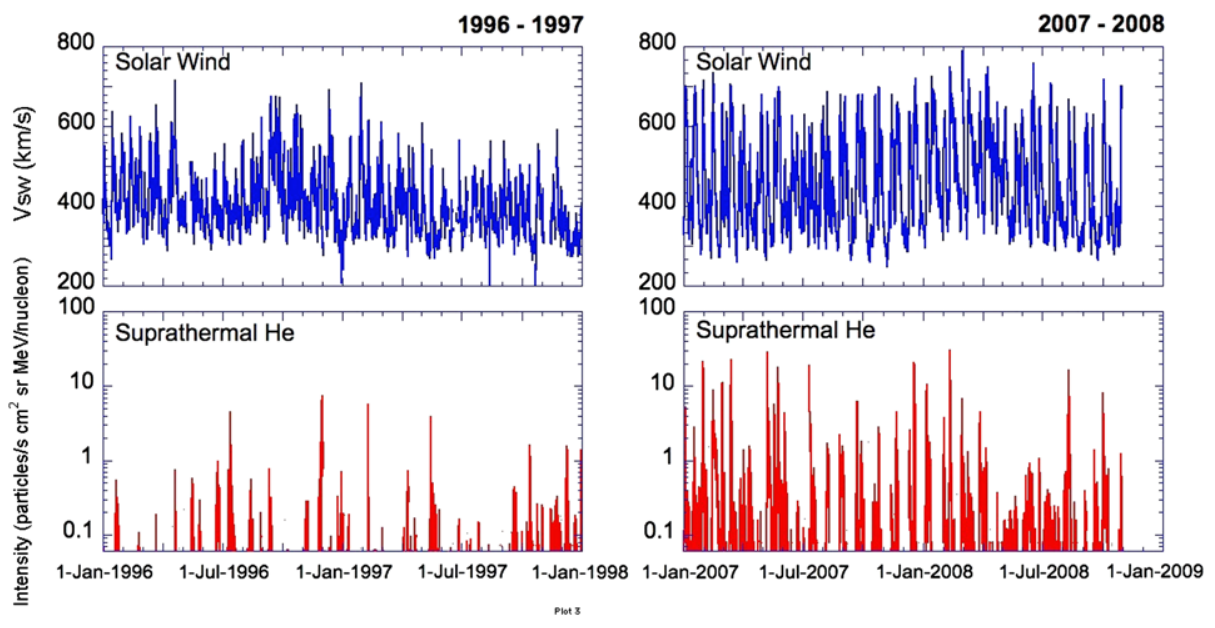

Figure 12 Hourly average Wind observations of solar wind protons (top panels) from the SWE instrument and suprathermal He (lower panels) from the EPACT/STEP instrument (left: 1996 - 1997, right: 2007 - 2008).

CIR \#2 begins with the appearance of a high speed solar wind stream, with a notable intensity drop around day 44.1 which lasts until nearly day 45.0 , when the intensity increases again. There is an additional drop for the period $\sim 46.3-46.5$. The spectrogram in panel (d) shows that the dropout timings are independent of energy. The first is associated with an increase in solar wind speed and the second with a decrease in solar wind speed. Although this CIR begins with a change of magnetic sector from outward to inward, the basic pointing of the magnetic field does not change markedly in association with these dropouts. The 2-point suprathermal spectral slope shown in panel (c) shows a significant steepening around day 44.0 (also visible in the spectrogram); thereafter it shows slow but systematic hardening as is typical for low energy CIR spectra (e.g., Mason et al., 1997) and is believed to be due to the inability of low energy ions to propagate from large radial distances in to $1 \mathrm{AU}$, so that the low energies are less abundant later in the event (see discussions in Fisk and Lee, 1980; Mason et al., 1999; Giacalone, Jokipii, and Kóta, 2002). The short-term decreases in CIR suprathermal intensity appear to be due to changes in connection to the CIR caused by the associated solar wind speed changes, which connect the 1 AU spacecraft to different locations in the region outside $1 \mathrm{AU}$. The fact that the dropout can occur with either an increase or decrease of solar wind speed, along with the energy independence of the intensity changes, and small effect on spectral index later in the event, indicates that this is a connection issue, and not caused by temporal changes in the CIR itself.

\section{Summary and Discussion}

During the 2007-2008 solar minimum period the STEREO spacecraft offered a new opportunity to study CIRs from spacecraft separated by up to five days of corotation. During this period there were numerous high speed solar wind streams and associated energetic particle increases from CIRs, with source locations at several longitudes on the Sun during 2007, simplifying to two source locations during 2008. Although all the energetic particle CIRs were associated with high speed streams, there were many streams that did not produce CIR events. Spectral forms of heavy nuclei in these CIRs were similar to those seen in earlier surveys with ACE and Wind; however, when compared with the ACE 1998-2006 


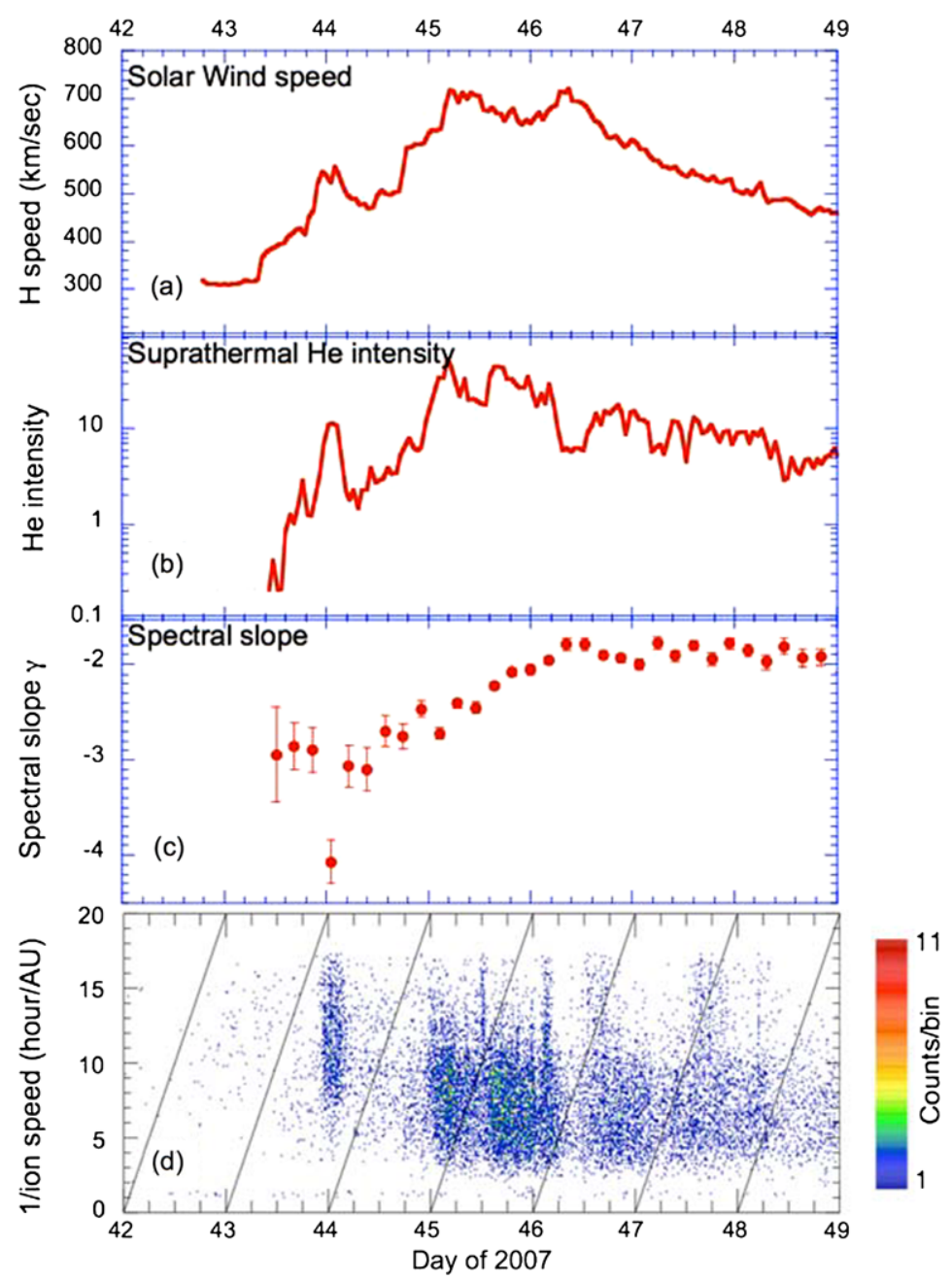

Figure $13 \mathrm{ACE}$ (a) solar wind speed, (b) $189 \mathrm{keV}$ nucleon $^{-1} \mathrm{He}$ intensity (particles/( $\mathrm{s} \mathrm{cm}^{2} \mathrm{srMeV}$ nucleon $\left.\left.^{-1}\right)\right)$, (c) 4-hour average suprathermal He spectral slope for intensity bins centered at 193 and $772 \mathrm{keV}$ nucleon $^{-1}$, and (d) ion arrival spectrogram for $\mathrm{C}$ and heavier elements, showing CIR "dropout" early on day 44 and at day 46.3. Brief low energy events (e.g., near day 45.5 or 46.1$)$ are upstream ions from the Earth's bow shock.

survey, the $2007-2008$ spectral slopes were slightly steeper ( $\sim 0.14$ units) below $1 \mathrm{MeV}$ per nucleon.

As the STEREO-A and -B spacecraft moved further from Earth, it was usually, but not always, possible to trace individual CIRs as they moved sequentially past STEREO-B to ACE and Wind, and then to STEREO-A. However it was often the case that events evolved significantly between one spacecraft and another: sometimes growing in intensity, sometimes decreasing. Even in cases where a clear event was seen at -B, Earth, and -A, the energetic particle events onsets sometimes varied from the expected corotation time; as an example, the CIR event starting at ACE on 2008 day $~ 222.0$ had its onset at STEREO-A near day 224.0, almost a full day earlier than would be expected from corotation. In addition 
to such simple changes, sometimes the overall time-intensity profiles would change dramatically from one spacecraft to another. While there is some temporal evolution of the CIRs over periods of days, the fact that many of the events are recurring indicates that the overall structures can be present for periods of several solar rotations. For this reason, it is likely that the bulk of the changes we observe were due to the relatively small and irregular coronal hole source sizes at the Sun, taken together with the different heliolatitude connections of the spacecraft connecting to different solar features. Sanderson et al. (1998) discussed such effects in detail for Wind data, showing the importance of the solar connection point and large differences in energetic particle observations that could arise from small changes in connection to the Sun. Leske et al. (2008) have discussed these effects for higher energy CIR particles observed on STEREO.

The lack of nearly rigid corotation for CIRs has implications for "space weather" predictions for the onset of high-speed solar wind streams at Earth. It is well known that high speed solar wind streams energize the magnetosphere (e.g., Baker, 1995 and references therein) leading to enhanced radiation levels that can cause significant impacts on space hardware. To predict the onset of such streams accurately, a monitor located behind Earth at $1 \mathrm{AU}$ would see the streams before they impacted the magnetosphere, as STEREO-B has done. However, the suprathermal particle observations presented here imply that the onset time at Earth might be in error by a significant amount ( $\sim 1$ day) assuming simple corotation. It would be interesting to study this further using solar wind data and MHD models to see what accuracy could be achieved in predicting the stream onsets, intensities, and duration.

Over the period 1994-2008 suprathermal He observations in CIRs showed that these events are present for both solar active and solar minimum periods as has been seen earlier for higher energy particles (see Richardson et al., 1993 and references therein). During this period, the most intense events took place during periods of enhanced solar activity. These most intense CIRs may arise from a combination of circumstances, since no single feature (e.g., solar wind speed, coronal hole size, the presence of shocks at $1 \mathrm{AU}$, or presence of ambient suprathermal ions) separated this group from other less intense CIRs. The size distribution of CIRs shows a much clearer "cutoff" than seen for $10 \mathrm{MeV}$ SEP protons, while the SEP number distribution has an index of -0.4 , the CIRs cutoff is much sharper.

Comparing the 1996-1997 vs. 2007-2008 solar minimum years, the 2007-2008 period had much better defined solar wind stream episodes, and many solar wind streams exceeding $600 \mathrm{~km} \mathrm{~s}^{-1}$ in contrast to the 1996-1997 period. This produced many more CIRs over our threshold for $189 \mathrm{keV} \mathrm{nucleon}^{-1} \mathrm{He}$ : 37 in 2007-2008 vs. 14 in $1996-1997$. For $386 \mathrm{keV}$ nucleon ${ }^{-1}$ hourly peak intensities above 10 particles/( $\mathrm{s} \mathrm{cm}^{2} \mathrm{sr} \mathrm{MeV}$ nucleon $\left.^{-1}\right)$, the $1996-1997$ period produced only one event, while the $2007-2008$ period produced 14 (through early November 2008).

About 25\% of the CIRs observed during 2007-2008 showed features in the $<1 \mathrm{MeV}$ nucleon $^{-1}$ energy range where the intensities decreased by a factor of $5-10$ for periods of a day or so in length ("dropout" events). These decreases occurred independent of particle energy, and therefore appear to be spatial rather than temporal effects. They correlate with changes in the solar wind speed, pointing to changes in connection to the distant CIR beyond $1 \mathrm{AU}$ as the primary cause. Because many of the originating solar coronal holes for the CIRs in this survey were small and irregularly shaped, it appears probable that these dropouts are caused by the spacecraft moving through different solar wind speed regions as the connection point at the Sun moves across irregular boundaries of the coronal hole, causing changes in connection to the distant CIR beyond 1 AU.

Acknowledgements We thank the Wind/SWE team for solar wind speed data used here and the ACE/MAG team for their list of transients and disturbances maintained on the ACE Science Center web site. Figure 8 
was produced using the Solar Weather Browser, sponsored by the Royal Observatory of Belgium and ESA. Sunspot numbers in Figure 10, and SEP event sizes in Figure 11 were obtained from the NOAA ftp site. The work at the Johns Hopkins University/Applied Physics Laboratory was supported by NASA under grant NNX07AP69G and contract SA4889-26309 from the University of California Berkeley. This work was also supported by the Max-Planck-Gesellschaft zur Foerderung der Wissenschaften and the Bundesministerium für Bildung und Forschung (BMBF) under grant 50 OC 0501.

Open Access This article is distributed under the terms of the Creative Commons Attribution Noncommercial License which permits any noncommercial use, distribution, and reproduction in any medium, provided the original author(s) and source are credited.

\section{References}

Baker, D.N.: 1995, Surv. Geophys. 16, 331

Desai, M.I., Marsden, R.G., Sanderson, T.R., Balogh, A., Forsyth, R.J., Gosling, J.T.: 1998, J. Geophys. Res. 103, 2003.

Fisk, L.A., Jokipii, J.R.: 1999, Space Sci. Rev. 89, 115.

Fisk, L.A., Lee, M.A.: 1980, Astrophys. J. 237, 620.

Forsyth, R.J., Marsch, E.: 1999, Space Sci. Rev. 89, 7.

Galvin, A.B., Kistler, L.M., Popecki, M.A., Farrugia, C.J., Simunac, K.D.C., Ellis, L., Möbius, E., Lee, M.A., Boehm, M., Carroll, J., et al.: 2008, Space Sci. Rev. 136, 437.

Giacalone, J., Jokipii, J.R., Kóta, J.: 2002, Astrophys. J. 573, 845.

Gloeckler, G.: 1999, Space Sci. Rev. 89, 91.

Gloeckler, G., Hovestadt, D., Fisk, L.A.: 1979, Astrophys. J. 230, L191.

Gopalswamy, N., Yashiro, S., Michalek, G., Kaiser, M.L., Howard, R.A., Reames, D.V., Leske, R.L., von Rosenvinge, T.T.: 2002, Astrophys. J. 72, L103.

Gosling, J.T., Pizzo, V.J.: 1999, Space Sci. Rev. 89, 21.

Horbury, T.S., Schmidt, J.M.: 1999, Space Sci. Rev. 89, 61.

Jian, L., Russell, C.T., Luhmann, J., Skoug, R.M.: 2006, Solar Phys. 239, 337.

Leske, R.A., Mewaldt, R.A., Mason, G.M., Cohen, C.M.S., Cummings, A.C., Davis, A.J., Labrador, A.W., Miyasaka, H., Stone, E.C., Wiedenbeck, M.E., et al.: 2008, In: Li, G., Hu, Q., Verkhoglyadova, O., Zank, G.P., Lin, R.P., Luhmann, J. (eds.) Particle Acceleration and Transport in the Heliosphere and Beyond: 7th Annual International Astrophysics Conf., AIP Conf. Proc. 1039, 131.

Mason, G.M., Sanderson, T.R.: 1999, Space Sci. Rev. 89, 77.

Mason, G.M., Mazur, J.E., Dwyer, J.R., Reames, D.V., von Rosenvinge, T.T.: 1997, Astrophys. J. 486, L149.

Mason, G.M., Gold, R.E., Krimigis, S.M., Andrews, J.E.M.B., Daley, K.A., Dwyer, J.R., Heuerman, K.F., James, T.L., Kennedy, M.J., LeFevere, T., et al.: 1998, Space Sci. Rev. 86, 409.

Mason, G.M., von Steiger, R., Decker, R.B., Desai, M.I., Dwyer, J.R., Fisk, L.A., Gloeckler, G., Gosling, J.T., Hilchenbach, M., Kallenbach, R., et al.: 1999, Space Sci. Rev. 89, 327.

Mason, G.M., Korth, A., Walpole, P.H., Desai, M.I., von Rosenvinge, T.T., Shuman, S.A.: 2008a, Space Sci. Rev. 136, 257.

Mason, G.M., Leske, R.A., Desai, M.I., Cohen, C.M.S., Dwyer, J.R., Mazuk, S., Mewaldt, R.A., Gold, R.E., Krimigis, S.M.: 2008b, Astrophys. J. 678, 1458.

McComas, D.J., Bame, S.J., Barker, P., Feldman, W.C., Phillips, J.L., Riley, P., Griffee, J.W.: 1998, Space Sci. Rev. 86, 563.

McCracken, K.G., Dreschhoff, G.A.M., Zeller, E.J., Smart, D.F., Shea, M.A.: 2001, J. Geophys. Res. 106, 21585.

Ogilvie, K.W., Chornay, D.J., Fritzenreiter, R.J., Hunsaker, F., Keller, J., Lobell, J., Miller, G., Scudder, J.D., Sittler, E.C. Jr., Torbert, R.B., et al.: 1995, Space Sci. Rev. 71, 55.

Reedy, R.C.: 1996, In: Balasubrahmanyan, K.S., Kiel, S.L., Smartt, R.N. (eds.) Solar Drivers of Interplanetary and Terrestrial Disturbances CS-95, Astron. Soc. Pac., San Francisco, 429.

Richardson, I.G.: 2004, Space Sci. Rev. 111, 267.

Richardson, I.G., Barbier, L.M., Reames, D.V., von Rosenvinge, T.T.: 1993, J. Geophys. Res. $98,13$.

Sanderson, T.R., Lin, R.P., Larson, D.E., McCarthy, M.P., Parks, G.K., Bosqued, J.M., Lormant, N., Ogilvie, K., Lepping, R.P., Szabo, A., et al.: 1998, J. Geophys. Res. 103, 17235.

Scholer, M.: 1999, Space Sci. Rev. 89, 105.

Simnett, G.M., Sayle, K.A., Roelof, E.C., Tappin, S.J.: 1994, Geophys. Res. Lett. 21, 1561.

Van Hollebeke, M.A.I., Ma Sung, L.S., McDonald, F.B.: 1975, Solar Phys. 41, 189.

von Rosenvinge, T.T., Barbier, L.M., Karsch, J., Liberman, R., Madden, M.P., Nolan, T., Reames, D.V., Ryan, L., Singh, S., Trexel, H., et al.: 1995, Space. Sci. Rev. 71, 155. 